\title{
On the Control of Two-link Flexible Robot Arm with Nonuniform Cross Section
}

\author{
MUSTAFA DOG̃AN \\ Department of Electrical and Electronics Engineering, Bașent University, Turkey \\ (mudogan@baskent.edu.tr) \\ ÖMER MORGÜL \\ Department of Electrical and Electronics Engineering, Bilkent University, Ankara, Turkey \\ (morgul@bilkent.edu.tr)
}

(Received 3 July 2008; accepted 21 April 2009)

\begin{abstract}
We consider the motion of a two-link flexible arm with nonuniform cross section. We obtain the equations of motion by using the extended Hamilton's principle. These equations consist of coupled partial differential equations and (nonlinear) ordinary differential equations with appropriate boundary conditions. Our control problem is to achieve the given desired link angles and suppress the link vibrations. To solve this problem, we propose a novel control scheme which consists of a dominant control law together with a parallel controller. We show that with the proposed controller, the control objectives are satisfied. Our stability analysis is based on the Lyapunov approach and LaSalle's invariance principle extended to infinite-dimensional systems. We also present some simulation results, which indicate that large parameter uncertainties such as tip and hub mass changes are also handled effectively by the proposed controller.
\end{abstract}

Key Words: Partial differential equations, flexible systems, nonuniform beams, boundary control.

\section{NOMENCLATURE}

$\begin{array}{ll}E & \text { Young's modulus } \\ I_{i}\left(x_{i}\right) & \text { Variable beam cross section moment about the } z \text {-axis at the location } x_{i} \\ I_{\mathrm{h} i} & \text { Inertia of } i \text { th hub } \\ I_{\mathrm{t} i} & \text { Tip inertia of } i \text { th beam } \\ l_{i} & \text { Length of } i \text { th link } \\ m_{\mathrm{h} i} & \text { Mass of } i \text { th hub } \\ m_{\mathrm{t} i} & \text { Tip mass of } i \text { th beam } \\ t & \text { Time }(t \geq 0) \\ x_{i} & \text { Coordinate along the axial center of } i \text { th beam }\left(0 \leq x_{i} \leq l_{i}\right) \\ w_{i}\left(x_{i}, t\right) & \text { Flexural deflection of point } i \text { at the location } x_{i} \text { of } i \text { th beam } \\ \dot{w}_{i}\left(x_{i}, t\right) & \text { Time rate of transverse movement of point } i \text { at the location } x_{i}, \\ & \text { where a dot represents time derivative }\end{array}$




$\begin{array}{ll}w_{i x}\left(x_{i}, t\right) & \begin{array}{l}\text { Flexural slope of point } i \text { at the location } x_{i}, \\ \text { where the subscript in } w_{i x} \text { denotes spatial derivative w.r.t. } x\end{array} \\ w_{i x x}\left(x_{i}, t\right) & \begin{array}{l}\text { Bending strain of point } i \text { at the location } x_{i} \\ \text { the base strain measured torques for the first and second beams }\end{array} \\ \tau_{\mathrm{b} i} & \begin{array}{l}\text { the end strain measured torques for the first and second beams } \\ \tau_{\mathrm{e} i}\end{array} \\ \rho_{i}\left(x_{i}\right) & \text { Variable density of the } i \text { th link depending on the cross-sectional area } \\ b_{i}(x) & \text { Variable height of the links due to tapering } \\ a_{1} & \text { Linear slope for tapering } \\ \tau_{i} & \text { Input torque at } i \text { th motor } \\ \theta_{1} & \text { Angular position of the first link } \\ \widehat{\theta}_{2} & \text { Angular position of the second link } \\ \theta_{2} & \theta_{2}=\widehat{\theta}_{2}+w_{1 x}\left(l_{1}, t\right) \\ \theta_{1 \mathrm{~d}} & \text { Desired angular position of the first link } \\ \theta_{2 \mathrm{~d}} & \text { Desired angular position of the second link } \\ \exp & \text { Exponential function, i.e. exp }(z)=e^{z} \\ \mathrm{PDE} & \text { Partial differential equation } \\ \text { ODE } & \text { Ordinary differential equation } \\ \text { FEM } & \text { Finite-element method }\end{array}$

\section{INTRODUCTION}

Two main advantages of flexible robot arms are less weight and low energy consumption. However, the structural modeling and the control design of the flexible arms are much more complicated due to nonlinear coupling between elastic and rigid modes during complex maneuvers, especially with high angular velocities. Various methods have been proposed for control of flexible-link manipulators in the literature. Hybrid control of a single flexiblelink manipulator using feedback linearization and a singular perturbation approach has been used in Vandegrift et al. (1994). Adaptive feedback linearization has been applied successfully for a nonlinear discrete-time model of a single-link flexible manipulator; see e.g. Rokui and Khorasani (1994). Singular perturbation theory has also been used for position and force control in Siciliano et al. (2001). Strain feedback and active vibration control, see e.g. Zhang et al. (2002) and Raab et al. (1998), are other approaches. In order to improve the important features of flexible links such as low mass, low moments of inertia and high natural frequencies, optimal shape design can be used; see e.g. Moallem et al. (2000). Besides, the integrated structure control for nonuniform flexible links can be improved; see e.g. Zhu et al. (2001). Furthermore, a high fundamental frequency is desired, since it implies a large bandwidth that will allow for fast motion without causing serious vibration problems; see e.g. Wang et al. (1992).

In this paper, inspired by the approaches given in Moallem et al. (2000), Zhu et al. (2001) and Wang et al. (1992), we consider the motion of a two-link flexible arm with variable cross section. We first present the equations of motion, which are obtained by using the extended Hamilton's principle. These equations are nonlinear and contain coupled PDEs and ODEs with appropriate boundary conditions. Our control objectives are, given arbitrary desired link angles, to find appropriate control laws for the torques applied to the motors in order to 


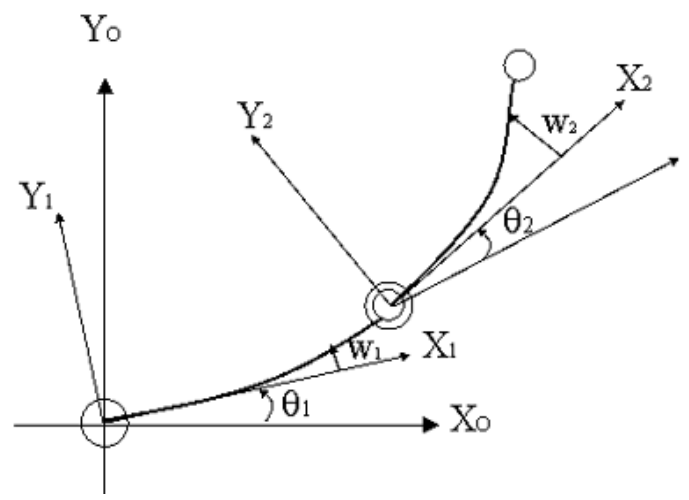

Figure 1. Arm configuration.

achieve the desired link angles as well as to suppress the link vibrations. We propose a novel control scheme which consists of a dominant controller augmented with a parallel controller. We show that the proposed controller achieves the control objectives. Our analysis is based on the Lyapunov approach applied to the original PDEs and LaSalle's invariance principle extended to infinite-dimensional systems. Hence, different from most of the control schemes proposed for multi-link flexible robots, see e.g. Wang et al. (2003, Ch. 2), we do not resort to modal truncation and similar methods to obtain a set of ODEs. It is well known that while the latter approach may lead to some stability and/or performance problems due to the neglected higher-order modes, our approach does not have such problems; see e.g. Luo et al. (1999).

The two-link flexible arm configuration which is considered in this paper is shown in Figure 1. Referring to Figure 1, the various symbols represent the following; $X_{0}, Y_{0}$ : global inertial system of coordinates; $X_{1}, Y_{1}$ : body-fixed system of coordinates attached to undeformed link $1, X_{2}, Y_{2}$ : body-fixed system of coordinates attached to undeformed link 2, $\theta_{1}, \theta_{2}$ : angular displacements of links 1 and $2, w_{1}, w_{2}$ : flexural displacements of links 1 and 2 .

This paper is organized as follows. In the next section we present the equations of motion for the system shown in Figure 1. In Section 3, we present our controller and some calculations which show the stability of the closed-loop system. In Section 4, we present some simulation results and in Section 5 we give some concluding remarks. The rigorous stability results are given in the form of appendices to improve the readability of the paper.

\section{ANALYTICAL MODEL}

In this section, we derive the equations of motion for the system shown in Figure 1 by using the extended Hamilton's principle; see e.g. Meirovitch (2001). Our approach is based on Zhang's work for uniform links; see e.g. Zhang (2001) and Zhang et al. (2002). Since the ratio between the length of the beam and its thickness is sufficiently large as proposed in Meirovitch (2001) and Reddy (1993), links can be modeled as Euler-Bernoulli beams, which can only be deformed in the flexural direction. The links are modeled in clamped- 
free configuration, since natural modes of the separated clamped-free links agree very well with actual ones compared to pinned-free configuration; see e.g. Hastings et al. (1987). Assuming that the manipulator moves in the horizontal plane, in the absence of gravity the potential energy depends only on the flexural deflections.

In order to derive the PDE model, we first derive the kinetic and potential energy expressions for the links and hubs; see Appendix C. Note that the offsets $O_{\mathrm{h} i}, O_{\mathrm{t} i}$ shown in Figure 1 will not be considered in the following development due to modern design techniques of DC motors. By using the extended Hamilton's principle, we obtain

$$
\int_{t_{1}}^{t_{2}}\left(\delta T-\delta V_{\mathrm{s}}+\delta W_{\mathrm{nc}}\right) d t=0,
$$

where $\delta T$ and $\delta V_{\mathrm{s}}$ are the variations of total kinetic and potential energies, respectively, and $\delta W_{\mathrm{nc}}$ is the variation of nonconservative work; see e.g. Meirovitch (2001, Section 6.4). Thus, after some straightforward but lengthy calculations we obtain the governing equations for a two-link flexible arm with nonuniform cross section by the variational method and integration by parts. These equations are listed below:

$$
\begin{aligned}
& \ddot{w}_{1}+\frac{\left(E I_{1}\left(x_{1}\right) w_{1 x x}\right)_{x x}}{\rho_{1}\left(x_{1}\right)}=-x_{1} \ddot{\theta}_{1}, \\
& \ddot{w}_{2}+\frac{\left(E I_{2}\left(x_{2}\right) w_{2 x x}\right)_{x x}}{\rho_{2}\left(x_{2}\right)}+\cos \theta_{2}\left[\ddot{w}_{1}\left(l_{1}, t\right)+l_{1} \ddot{\theta}_{1}\right] \\
- & \dot{\theta}_{2} \sin \theta_{2}\left[\dot{w}_{1}\left(l_{1}, t\right)+l_{1} \dot{\theta}_{1}\right]=-x_{2} \ddot{\theta}_{2}, \\
& I_{h 1} \ddot{\theta}_{1}-E I_{1}(0) w_{1 x x}(0, t)=\tau_{1}, \\
& I_{t 1} I_{h 2} \ddot{\theta}_{2}-I_{h 2} E I_{1}\left(l_{1}\right) w_{1 x x}\left(l_{1}, t\right)-I_{t 1} E I_{2}\left(l_{2}\right) w_{2 x x}\left(l_{2}, t\right)=\left(I_{t 1}+I_{h 2}\right) \tau_{2}, \\
& -\dot{\theta}_{2} \sin \theta_{2}\left[\dot{w}_{1}\left(l_{1}, t\right)+l_{1} \dot{\theta}_{1}\right] \int_{0}^{l_{2}} \rho_{2}\left(x_{2}\right) x_{2} d x_{2}+\int_{0}^{l_{2}} x_{2}\left(E I_{2}\left(x_{2}\right) w_{2 x x}\right)_{x x} d x_{2} \\
- & I_{h 2}\left[\ddot{\theta}_{1}+\ddot{\theta}_{2}\right]-I_{t 2}\left[\ddot{\theta}_{1}+\ddot{\theta}_{2}+\ddot{w}_{2 x}\left(l_{2}, t\right)\right] \\
- & \sin \theta_{2}\left[\dot{w}_{1}\left(l_{1}, t\right)+l_{1} \dot{\theta}_{1}\right] \int_{0}^{l_{2}} \rho_{2}\left(x_{2}\right) \dot{w}_{2} d x_{2} \\
- & m_{t 2} l_{2}\left[l_{2} \ddot{\theta}_{2}+\ddot{w}_{2}\left(l_{2}, t\right)+\cos \theta_{2}\left[\ddot{w}_{1}\left(l_{1}, t\right)+l_{1} \ddot{\theta}_{1}\right]\right] \\
- & m_{t 2} \sin \theta_{2}\left[\dot{w}_{1}\left(l_{1}, t\right)+l_{1} \dot{\theta}_{1}\right] \dot{w}_{2}\left(l_{2}, t\right) \\
- & \left.I_{t 1} \ddot{\theta}_{1}+\ddot{w}_{1 x}\left(l_{1}, t\right)\right]-E I_{1}\left(l_{1}\right) w_{1 x x}\left(l_{1}, t\right)=0,
\end{aligned}
$$




$$
\begin{aligned}
& -\int_{0}^{l_{2}} \rho_{2}\left(x_{2}\right)\left\{\left[\ddot{w}_{1}\left(l_{1}, t\right)+l_{1} \ddot{\theta}_{1}\right]+\cos \theta_{2}\left[\ddot{w}_{2}+x_{2} \ddot{\theta}_{2}\right]\right. \\
& \left.-\quad \dot{\theta}_{2} \sin \theta_{2}\left[\dot{w}_{2}+x_{2} \dot{\theta}_{2}\right]\right\} d x_{2}-\left(m_{t 1}+m_{h 2}+m_{t 2}\right)\left[\ddot{w}_{1}\left(l_{1}, t\right)+l_{1} \ddot{\theta}_{1}\right] \\
& +\quad\left[\left(E I_{1}\left(x_{1}\right) w_{1 x x}\right)_{x}\right]_{x_{1}=l_{1}}-m_{t 2}\left\{\cos \theta_{2}\left[\ddot{w}_{2}\left(l_{2}, t\right)+l_{2} \ddot{\theta}_{2}\right]\right. \\
& \left.-\quad \dot{\theta}_{2} \sin \theta_{2}\left[\dot{w}_{2}\left(l_{2}, t\right)+l_{2} \dot{\theta}_{2}\right]\right\}=0, \\
& \quad\left[\left(E I_{2}\left(x_{2}\right) w_{2 x x}\right)_{x}\right]_{x_{2}=l_{2}} m_{t 2}\left\{\left[\ddot{w}_{2}\left(l_{2}, t\right)+l_{2} \ddot{\theta}_{2}\right]+\cos \theta_{2}\left[\ddot{w}_{1}\left(l_{1}, t\right)+l_{1} \ddot{\theta}_{1}\right]\right. \\
& \left.-\quad \dot{\theta}_{2} \sin \theta_{2}\left[\dot{w}_{1}\left(l_{1}, t\right)+l_{1} \dot{\theta}_{1}\right]\right\}=0, \\
& I_{t 2}\left[\ddot{\theta}_{1}+\ddot{\theta}_{2}+\ddot{w}_{2 x}\left(l_{2}, t\right)\right]+E I_{2}\left(l_{2}\right) w_{2 x x}\left(l_{2}, t\right)=0, \\
& w_{1}(0, t)=w_{1 x}(0, t)=w_{2}(0, t)=w_{2 x}(0, t)=0,
\end{aligned}
$$

where all offset values $\left(O_{\mathrm{h} i}, O_{\mathrm{t} i}\right)$ are neglected as in Zhang (2001) and Zhang et al. (2002). Moreover, as in Zhang (2001) and Zhang et al. (2002), since $w_{1 x}\left(l_{1}, t\right)$ is relatively small, this term is also neglected; hence, we assume that $\theta_{2}=\widehat{\theta}_{2}$. For details, see e.g. Zhang (2001) and Zhang et al. (2002).

The equations 2 and 3 are the main PDEs for the flexible links and the equations 4 and 5 are ODEs for rigid bodies. Equations (6-10) are the boundary conditions, where equation 10 includes the four boundary conditions at the clamped ends of the links; see e.g. Hartog (1985).

We note that these equations can be obtained by using equation 1 , the extended Hamilton's principle, see e.g. Meirovitch (2001, Section 6.4); the related expressions are given in Appendix C. The required calculations are rather straightforward but lengthy, and hence are omitted here to improve the readability of the paper. For similar calculations for the uniform link case, see e.g. Zhang (2001); Zhang et al. (2002).

We first define the following variables for $i=1,2$ :

$$
\begin{aligned}
\tau_{b i} & =-E I_{i}(0) w_{i x x}(0, t), \\
\tau_{e i} & =-E I_{i}\left(l_{i}\right) w_{i x x}\left(l_{i}, t\right),
\end{aligned}
$$

where $\tau_{b 1}, \tau_{b 2}$ represent the base strain measured torques and $\tau_{e 1}, \tau_{e 2}$ represent the end strain measured torques for the first and second links, respectively. Boundary conditions 6,8 and 9 can be simplified further to obtain the following: 


$$
\begin{gathered}
\sin \theta_{2}\left[\dot{w}_{1}\left(l_{1}, t\right)+l_{1} \dot{\theta}_{1}\right]\left\{-\dot{\theta}_{2} \int_{0}^{l_{2}} \rho_{2}\left(x_{2}\right) x_{2} d x_{2}\right. \\
\left.-l_{2} \dot{\theta}_{2} m_{t 2}-\int_{0}^{l_{2}} \rho_{2}\left(x_{2}\right) \dot{w}_{2} d x_{2}-m_{t 2} \dot{w}_{2}\left(l_{2}, t\right)\right\} \\
-\tau_{b 2}+\tau_{e 1}-I_{h 2}\left[\ddot{\theta}_{1}+\ddot{\theta}_{2}\right]-I_{t 1}\left[\ddot{\theta}_{1}+\ddot{w}_{1 x}\left(l_{1}, t\right)\right]=0 .
\end{gathered}
$$

Hence, the new set of boundary conditions will be equations 7, 8, 10 and 13 .

Remark 2.1. We note that multi-link flexible structures are considered in the literature, but the majority of those works do not rely on PDE models; instead, they rely on finitedimensional ODE approximation of PDE models by using e.g. assumed modes, FEMs, etc.; see e.g. Meirovitch (2001) and Wang et al. (2003). This approach has many disadvantages, as noted in the literature. First, the flexible and rigid coordinates can be decoupled in these approaches, which is questionable from the physical point of view. Secondly, although the flexible link equations are coupled in the PDE approach, see equations 2-10, and this is quite reasonable from the physical point of view, each link equation can be decoupled by using the boundary assumptions in ODE approaches; see e.g. Zhang et al. (2004). Thirdly, usually vibration modes are truncated by ignoring higher-order modes, and this may result in a wellknown problem called control and/or observation spillovers, which may cause instability in the original system; see e.g. Luo (1993). We note that these ODE methods can be easily used to model multi-link flexible structures due to the decoupling of links; see e.g. Zhang et al. (2004). Infinite-dimensional PDE models can also be extended to model multi-link structures, but this usually requires highly complex analysis, which may become prohibitive for the case of more than two links; see e.g. Zhang et al. (2004).

Remark 2.2. Since we have already neglected $w_{1 x}$, we have also omitted $\ddot{w}_{i x}$ in the final calculations. Note that such terms are also omitted in Zhang et al. (2005).

\section{CONTROLLER DESIGN}

To control the system given by equations $2-10$ we propose a novel control law, which consists of a dominant controller and a parallel controller to ensure the asymptotic stability of the closed-loop system. With this insight, our proposed controller has the following form:

$$
\begin{aligned}
\tau_{1} & =\tau_{11}+\gamma_{1} \tau_{12}, \\
\tau_{2} & =\tau_{21}+\gamma_{2} \tau_{22},
\end{aligned}
$$

where $\tau_{11}$ and $\tau_{21}$ denote the dominant part of the controller and $\tau_{12}$ and $\tau_{22}$ denote the parallel controller, which augments the dominant part of the controller. Here, $\gamma_{1}, \gamma_{2}>0$ 
are proportional gains which may be adjusted to make the control laws $\tau_{11}, \tau_{21}$ dominant. This can be achieved by choosing $\gamma_{1}$ and $\gamma_{2}$ sufficiently small. For the dominant part of the controller, we propose the following:

$$
\begin{aligned}
\tau_{11} & =\tau_{b 1}+I_{h 1}\left(\frac{-\ddot{w}_{1}\left(l_{1}, t\right)}{l_{1}}-K_{1}\left(\alpha \dot{\theta}_{1}+\beta\left(\theta_{1}-\theta_{1 d}\right)\right)\right), \\
\tau_{21} & =\left(I_{h 2} \tau_{e 1}+I_{t 1} \tau_{e 2}+I_{t 1} I_{h 2}\left(\frac{-\ddot{w}_{2}\left(l_{2}, t\right)}{l_{2}}-K_{2}\left(\alpha \dot{\theta}_{2}+\beta\left(\theta_{2}-\theta_{2 d}\right)\right)\right)\right) \\
& /\left(I_{t 1}+I_{h 2}\right),
\end{aligned}
$$

where $K_{1}, K_{2}, \alpha, \beta$ are positive constants and $\theta_{1 d}, \theta_{2 d}$ are the desired link angles. For the parallel controller, we propose the following:

$$
\begin{aligned}
\tau_{12} & =\tau_{b 2}-\tau_{e 2}-K \dot{\theta}_{1}, \\
\tau_{22} & =\left(I_{t 1} I_{h 2}\left(\ddot{\theta}_{1}+\ddot{\theta}_{2}\right)+I_{t 1} \tau_{e 2}\right) /\left(I_{t 1}+I_{h 2}\right),
\end{aligned}
$$

where $K>0$ is a proportional gain.

Since the system 2-10 became decoupled and linear by the dominant part of the controller, additivity and homogeneity properties of linear systems can be applied for the closedloop system; see Appendix A for necessary calculations. Thus, the effect of the control laws $\tau_{12}, \tau_{22}$ should be considered independently from the effect of the dominant control laws.

In the sequel, we present some necessary calculations related to our stability analysis. Since the proof of the asymptotic stability of the closed-loop system requires some rigorous definitions and some lengthy calculations, the main part of the proof is given in Appendix B to improve the readability of the paper.

Our approach is based on the Lyapunov stability analysis. As in most mechanical structures, the total energy of the system shown in Figure 1 together with a correction term may be used as a Lyapunov function candidate. Such a function $V$, which will be used in the rest of the paper, is given below:

$$
V=T_{1}+T_{2}+T_{3}+T_{4}+T_{5}+T_{6}+V_{s}+\ddot{\theta}_{1}^{2} \geq 0
$$

where $T_{i}, i=1, \ldots, 6$, are the kinetic energy terms of links, tips and hubs for links 1 and 2 . The total strain potential energy for both links is represented as $V_{s}$; see Appendix C. After applying the dominant control laws to the system 2-10, see Appendix A, and after some lengthy but straightforward calculations, the total energy rate of the system is obtained as follows:

$$
\dot{V}=\left(\tau_{e 2}-\tau_{b 2}+\tau_{b 1}+I_{h 1} \ddot{\theta}_{1}\right) \dot{\theta}_{1}+\left(\tau_{e 1}-I_{t 1} \ddot{\theta}_{1}\right) \dot{\theta}_{2}+2 \ddot{\theta}_{1} \frac{d \ddot{\theta}_{1}}{d t}
$$


By using equations 9 and 12, we easily obtain

$$
\tau_{e 2}=I_{t 2}\left(\ddot{\theta}_{1}+\ddot{\theta}_{2}\right)
$$

see Remark 2.2. By using equation 22 in equation 5 and then by using equations 12, 17, 19 and 15 , we obtain

$$
\frac{I_{t 2} I_{h 2} K_{2}}{\gamma_{2}\left(I_{h 2}+I_{t 2}\right)}\left[\alpha \dot{\theta}_{2}+\beta\left(\theta_{2}-\theta_{2 d}\right)\right]=\tau_{e 2} .
$$

By evaluating equation 3 at $x_{2}=l_{2}$, using equation 8, and Appendix A, it follows that $\tau_{e 2}$ and $\dot{\tau}_{e 2}$ satisfy the following inequalities:

$$
\left|\tau_{e 2}\right| \leq C_{e 2} \exp \left(-\delta_{e 2} t\right), \quad\left|\dot{\tau}_{e 2}\right| \leq \widehat{C}_{e 2} \exp \left(-\widehat{\delta}_{e 2} t\right)
$$

where $C_{e 2}, \widehat{C}_{e 2}, \delta_{e 2}, \widehat{\delta}_{e 2}$ are some positive constants; see also e.g. Baruh (1999, Section 11.4) and Hattori et al. (1997). Then, by using equation 24 in equation 23, one can easily obtain the following:

$$
\begin{aligned}
& \theta_{2}(t)=\theta_{2 d}-\theta_{2 d} \exp \left(-\frac{\beta}{\alpha} t\right)+f_{1}(t), \\
& \dot{\theta}_{2}(t)=\theta_{2 d} \frac{\beta}{\alpha} \exp \left(-\frac{\beta}{\alpha} t\right)+f_{2}(t), \\
& \ddot{\theta}_{2}(t)=-\theta_{2 d}\left(\frac{\beta}{\alpha}\right)^{2} \exp \left(-\frac{\beta}{\alpha} t\right)+f_{3}(t),
\end{aligned}
$$

where $f_{i}(t)$ are some exponentially decaying functions satisfying

$$
\left|f_{i}(t)\right| \leq C_{i} \exp \left(-\gamma_{i} t\right)
$$

for some positive constants $C_{i}, \gamma_{i}, i=1,2,3$.

By using equations 3, 8 and 25, it follows that, similar to equation 24, the measured base torque $\tau_{b 2}$ also satisfies the following:

$$
\left|\tau_{b 2}\right| \leq C_{b 2} \exp \left(-\delta_{b 2} t\right), \quad\left|\dot{\tau}_{b 2}\right| \leq \widehat{C}_{b 2} \exp \left(-\widehat{\delta}_{b 2} t\right),
$$

where $C_{b 2}, \widehat{C}_{b 2}, \delta_{b 2}, \widehat{\delta}_{b 2}$ are some positive constants; see also the discussion after equation 23, Baruh (1999, Section 11.4), Hattori et al. (1997) and Appendices A and B.

By using equations 4, 14, 16 and 18, and using Appendix A, we obtain

$$
K_{1}\left(\alpha \dot{\theta}_{1}+\beta\left(\theta_{1}-\theta_{1 d}\right)\right)+\frac{\gamma_{1}}{I_{h 1}} K \dot{\theta}_{1}=g(t)
$$


where $g(t)=\frac{\gamma_{1}}{I_{h 1}}\left(\tau_{b 2}-\tau_{e 2}\right)$. By using equations 24 and 27, it follows that $g(t)$ satisfies the following:

$$
|g(t)| \leq C_{g} \exp \left(-\delta_{g} t\right), \quad|\dot{g}(t)| \leq \widehat{C}_{g} \exp \left(-\widehat{\delta}_{g} t\right)
$$

for some positive constants $C_{g}, \widehat{C}_{g}, \delta_{g}, \widehat{\delta}_{g}$. Then, by using equation 29 in equation 28 , similar to equation 25 , one can easily obtain the following:

$$
\begin{aligned}
\theta_{1}(t) & =\theta_{1 d}-\theta_{1 d} \exp \left(-K^{\prime} t\right)+\widehat{f}_{1}(t), \\
\dot{\theta}_{1}(t) & =\theta_{1 d} K^{\prime} \exp \left(-K^{\prime} t\right)+\widehat{f}_{2}(t), \\
\ddot{\theta}_{1}(t) & =-\theta_{1 d} K^{\prime 2} \exp \left(-K^{\prime} t\right)+\widehat{f}_{3}(t), \\
\ddot{\theta}_{1} \frac{d \ddot{\theta}_{1}}{d t} & =-\theta_{1 d}^{2} K^{\prime 5} \exp \left(-2 K^{\prime} t\right)+\widehat{f}_{4}(t) .
\end{aligned}
$$

Here, $K^{\prime}$ is a positive constant given by

$$
K^{\prime}=\frac{K_{1} \beta I_{h 1}}{K_{1} \alpha I_{h 1}+\gamma_{1} K}
$$

and $\widehat{f_{i}}(t)$ are some exponentially decaying functions satisfying

$$
\left|\widehat{f}_{i}(t)\right| \leq \widehat{C}_{i} \exp \left(-\widehat{\gamma}_{i} t\right)
$$

for some positive constants $\widehat{C}_{i}, \widehat{\gamma}_{i}, i=1,2,3,4$; see equation 26 .

By using equation 32 in equation 2, by utilizing the techniques presented in Appendix B (see e.g. equations 73 and 75), it can be shown that, similar to equations 24 and 27, we obtain the following inequalities for the measured base torques $\tau_{b 1}$ and $\tau_{e 1}$ :

$$
\left|\tau_{e 1}\right| \leq C_{e 1} \exp \left(-\delta_{e 1} t\right), \quad\left|\tau_{b 1}\right| \leq C_{b 1} \exp \left(-\delta_{b 1} t\right)
$$

Here, $C_{e 1}, C_{b 1}, \delta_{e 1}, \delta_{b 1}$ are some positive constants; see also the discussion after equation 23, Baruh (1999, Section 11.4), Hattori et al. (1997) and Appendix B.

By using equations $24,25,26,27,32,35$ and 36 in equation 21 , we obtain

$$
\begin{aligned}
\dot{V} & =I_{h 1} \ddot{\theta}_{1} \dot{\theta}_{1}+2 \ddot{\theta}_{1} \frac{d \ddot{\theta}_{1}}{d t}+h(t) \\
& =-\left(I_{h 1}+2 K^{\prime 2}\right) \theta_{1 d}^{2} K^{\prime 3} \exp \left(-2 K^{\prime} t\right)+h(t),
\end{aligned}
$$

where $K^{\prime}$ is given by equation 34 and $h(t)$ is an exponentially decaying function satisfying

$$
|h(t)| \leq C_{h} \exp \left(-\gamma_{h} t\right)
$$


for some positive constants $C_{h}, \gamma_{h}$; see equation 26. Since the right-hand side of equation 37 is integrable, it follows that

$$
V(t) \leq M, \quad \forall t \geq 0,
$$

where $M>0$ is a constant depending on initial conditions. Moreover, from equation 37, it also follows that

$$
\lim _{t \rightarrow \infty} \dot{V}(t)=0 .
$$

We note that equation 39 shows that the solutions of the closed-loop system, if they exist, remain bounded, which can be used to prove the stability of the closed-loop system. On the other hand, equation 40 could be used to prove the asymptotic stability of the closedloop system, by employing LaSalle's invariance principle extended to infinite-dimensional systems. The proof of existence, uniqueness and asymptotic stability of the solutions for the closed-loop system requires some rather lengthy calculations, and is given in Appendix B to improve the readability of the paper.

\section{SIMULATION RESULTS}

\subsection{PDE Model Case 1}

The proposed control scheme is tested with the simulation program implemented in MATLAB. The PDEs are discretized in the space domain by the finite-difference method to obtain ODEs at each of the nodes. Then, the ODEs are solved numerically. Instead of dealing with the complexity of the fourth order derivative approximation, the second order derivative approximation has been used with the help of auxiliary states. Those states are more meaningful in a real problem as well, since they correspond to physical variables such as deflections, velocities and bending moments; see e.g. Abhyankar et al. (1993). However, the number of ODEs to solve and the computation time are increased as a trade-off of the robust stability of the numerical scheme. The parameters used in the model for the system 2-10 are listed in Table 1; see Remark 4.1. The simulation results for PDE case 2 are presented in Figures 2-7.

\subsection{PDE Model Case 2}

In the second part of the simulations as PDE case 2, the proportional gain, $K$, in the parallel controller given by equation 18 has been increased from $K=9$ to $K=21$. To test the robustness to parameter changes, tip mass $m_{t 1}$ for link 1 and tip mass $m_{t 2}$ and hub mass $m_{h 2}$ for link 2 have been changed at the fifth second; see Table 2. The simulation results for PDE case 2 are presented in Figures 8-15. The step changes in masses did not affect the performance of the controller very much, as can be seen from the simulation results.

We observe that tip deflections of links become smaller by increasing $K$, as time responses exhibit smoother transitions than the ones for the PDE case 1. 
Table 1. Parameters of the flexible arm.

\begin{tabular}{ll}
\hline Parameter & Value \\
\hline Length of links & $l_{1}=0.5 \mathrm{~m}, l_{2}=0.6 \mathrm{~m}$ \\
Time step & $\Delta t=3 e-5 \mathrm{sec}$ \\
Spatial steps & $\Delta x_{1}=l_{1} / 20, \Delta x_{2}=l_{2} / 20$ \\
Young's modulus, $E$ & $70 \quad \mathrm{GPa}$ \\
Density, $\rho$ & $2742 \quad \mathrm{~kg} \mathrm{~m}^{-3}$ \\
Thickness of links (m) & $c_{1}=0.003175, c_{2}=0.00238$ \\
Maximum height for tapering & $b_{0}=0.0654 \mathrm{~m}$ \\
at the root of the link & \\
Linear slope for tapering & $a_{1}=0.04$ \\
Hub inertias $\left(\mathrm{kg} \mathrm{m}^{2}\right)$ & $I_{h 1}=0.0055, I_{h 2}=0.0068$ \\
Tip inertias $\left(\mathrm{kg} \mathrm{m}^{2}\right)$ & $I_{t 1}=0.0139, I_{t 2}=0.00024$ \\
Hub mass $(\mathrm{kg})$ & $m_{h 2}=0.678$ \\
Tip mass $(\mathrm{kg})$ & $m_{t 1}=0.981, m_{t 2}=0.204$ \\
$\theta_{1 d}$ (desired) & $\pi / 2 \quad \mathrm{rad}$ \\
$\theta_{2 d}$ (desired) & $-\pi / 2 \quad \mathrm{rad}$ \\
$K_{1}=0.007, \quad K_{2}=0.01$ & $\alpha=600, \quad \beta=800$ \\
$\gamma_{1}=1 e-3, \quad \gamma_{2}=1 e-4$ & $K=9$ \\
\hline
\end{tabular}

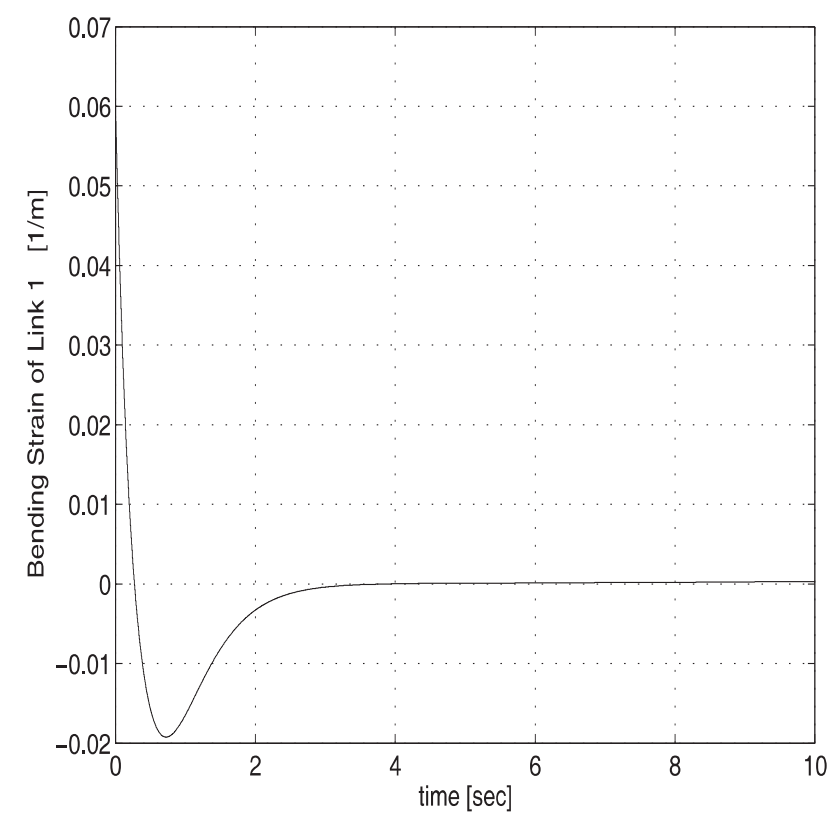

Figure 2. Bending strain at the end of the link 1. 
630 M. DOG̃AN and Ö. MORGÜL

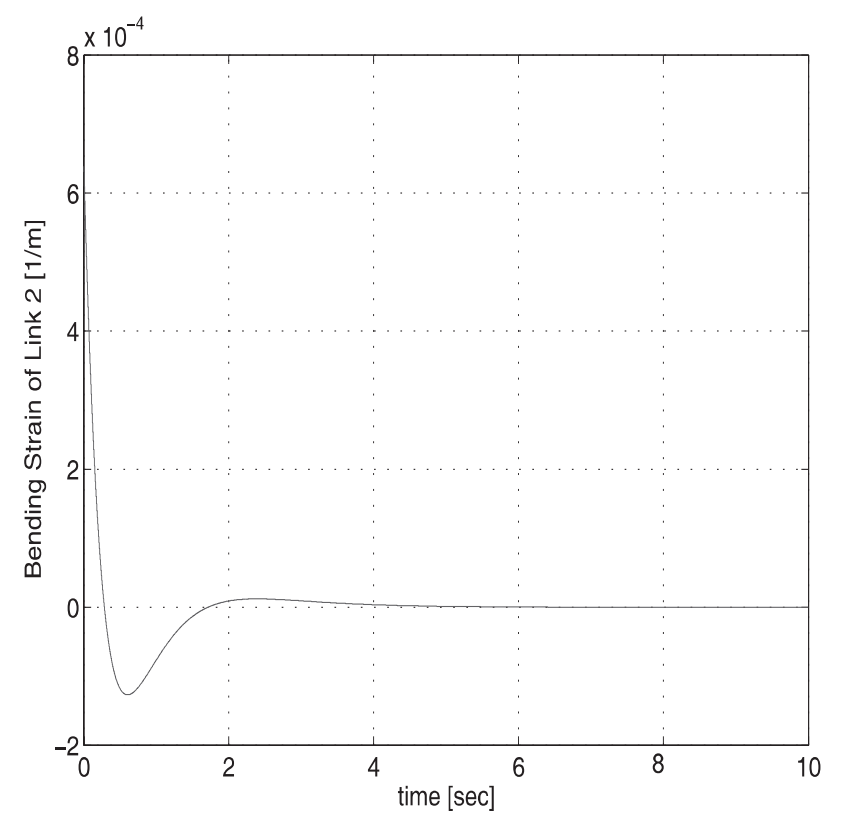

Figure 3. Bending strain at the end of the link 2.

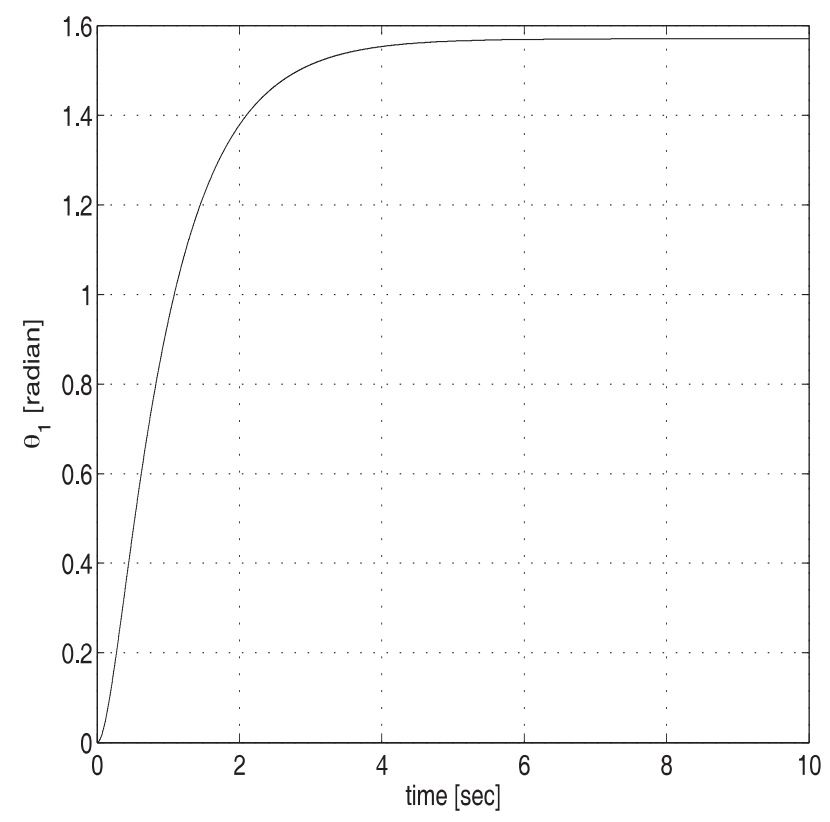

Figure 4. Joint angle of the link 1. 
ON THE CONTROL OF TWO-LINK FLEXIBLE ROBOT ARM 631

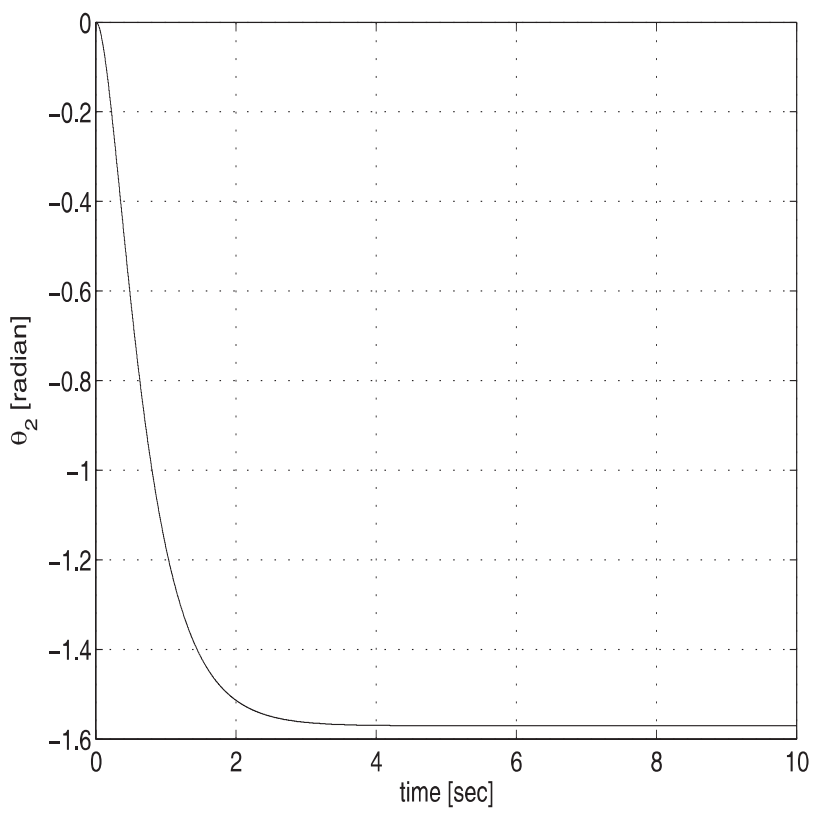

Figure 5. Joint angle of the link 2.

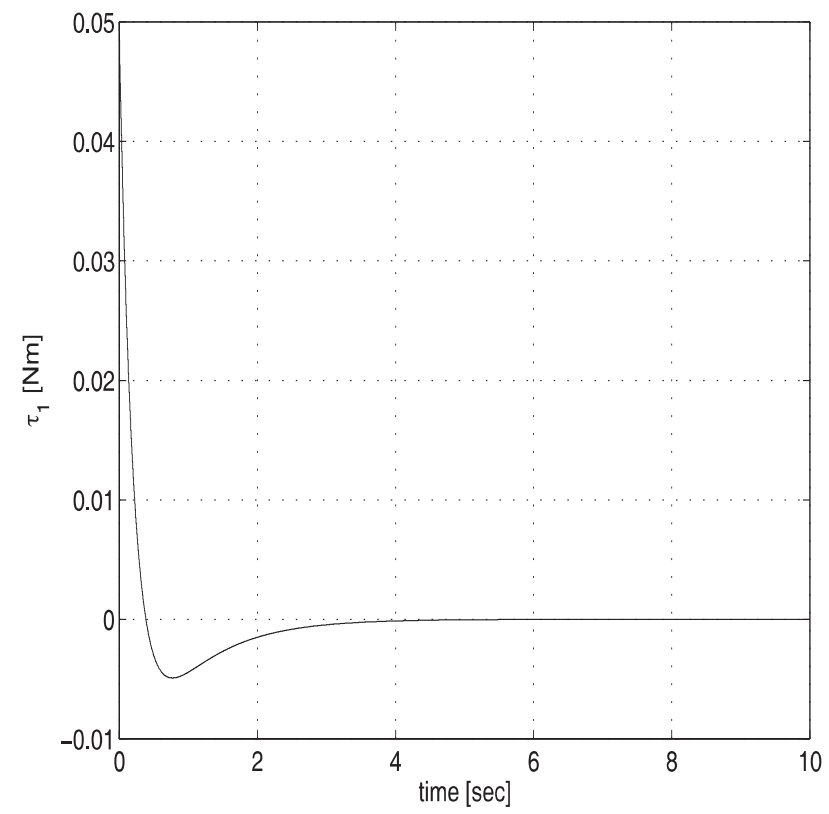

Figure 6 . Control torque of joint 1. 


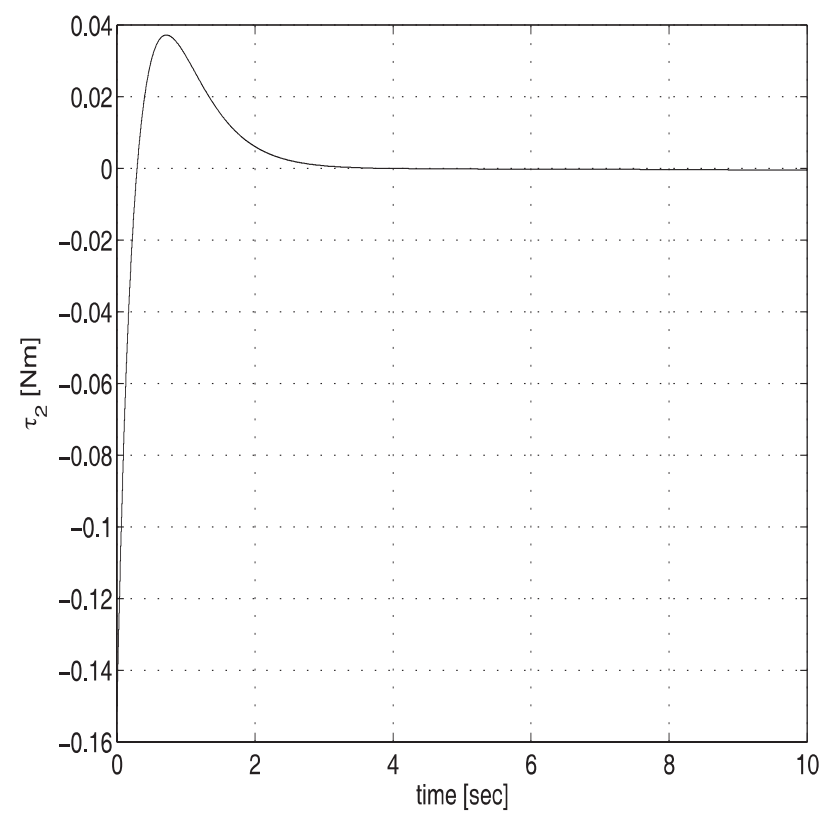

Figure 7. Control torque of joint 2.

Table 2. Tip and hub mass variation for PDE case 2.

\begin{tabular}{lllll}
\hline Parameter & & & Value \\
\hline Hub mass & $(0-5 \mathrm{sec})$ & $(\mathrm{kg})$ & $m_{h 2}=0.678$ & \\
Tip mass & $(0-5 \mathrm{sec})$ & $(\mathrm{kg})$ & $m_{t 1}=0.981$, & $m_{t 2}=0.204$ \\
Hub mass & $(5-10 \mathrm{sec})$ & $(\mathrm{kg})$ & $m_{h 2}=0.378$ & \\
Tip mass & $(5-10 \mathrm{sec})$ & $(\mathrm{kg})$ & $m_{t 1}=0.481$, & $m_{t 2}=0.704$ \\
\multicolumn{2}{l}{\begin{tabular}{l} 
Proportional gain \\
\hline
\end{tabular}} & & & \\
\hline
\end{tabular}

Although the control setup in the previous section is given for links that can have any kind of variable cross section, in this particular simulation rectangular cross sections of given uniform thickness are used. For small values of tip mass and tip inertia moment relative to the ones for a beam, the optimum shape is approximately a linearly tapered beam; see e.g. Wang et al. (1992). Therefore, the height $b_{i}(x)$, density $\rho_{i}(x)$ and cross-sectional area moment $I_{i}(x)$ at any point can be calculated with the parameters given in Table 1, such as

$$
\begin{aligned}
b_{i}(x) & =b_{0}-2 l_{i} a_{1}+2\left(l_{i}-x\right) a_{1}, \\
\rho_{i}(x) & =\rho b_{i}(x) c_{i}, \\
I_{i}(x) & =b_{i}(x) c_{i}^{3} / 12 .
\end{aligned}
$$

The simulation results are presented in Figures 2-7. Smooth time histories of all variables of interest without overshoot show the effectiveness of the controller performance especially with such demanding desired positions $\left(\theta_{1 d}=\pi / 2\right.$ and $\left.\theta_{2 d}=-\pi / 2\right)$. 


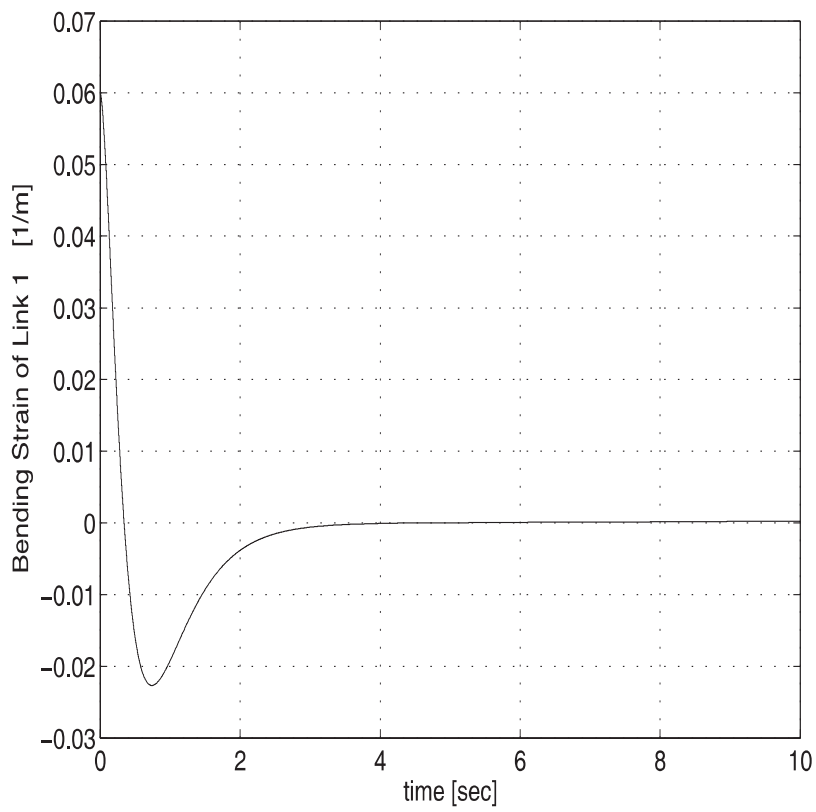

Figure 8 . Bending strain at the end of the link $1-$ PDE model case 2.

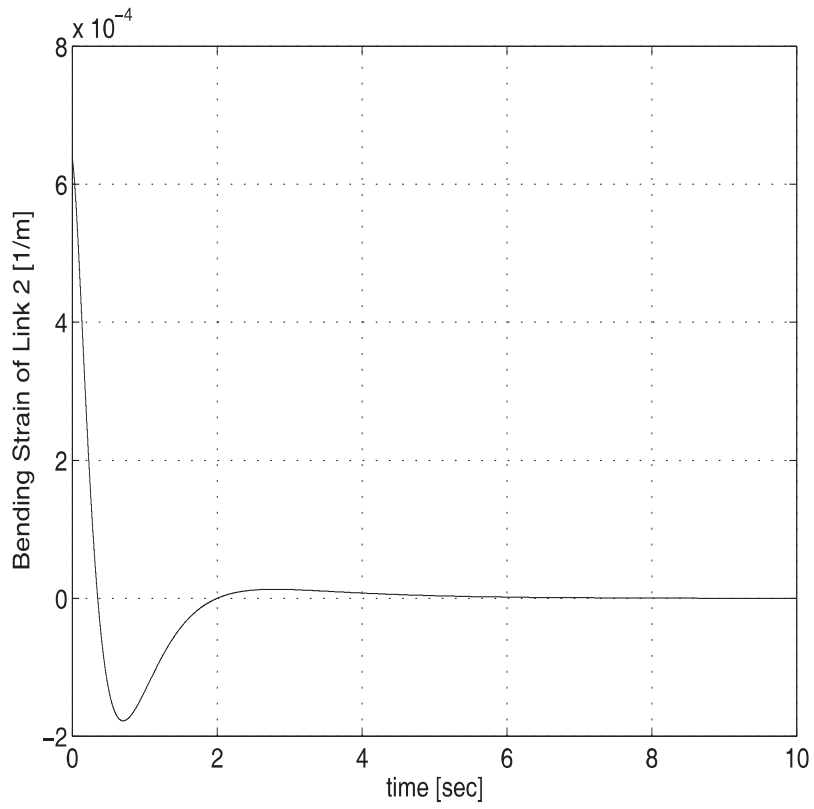

Figure 9. Bending strain at the end of the link $2-$ PDE model case 2. 
634 M. DOG̃AN and Ö. MORGÜL

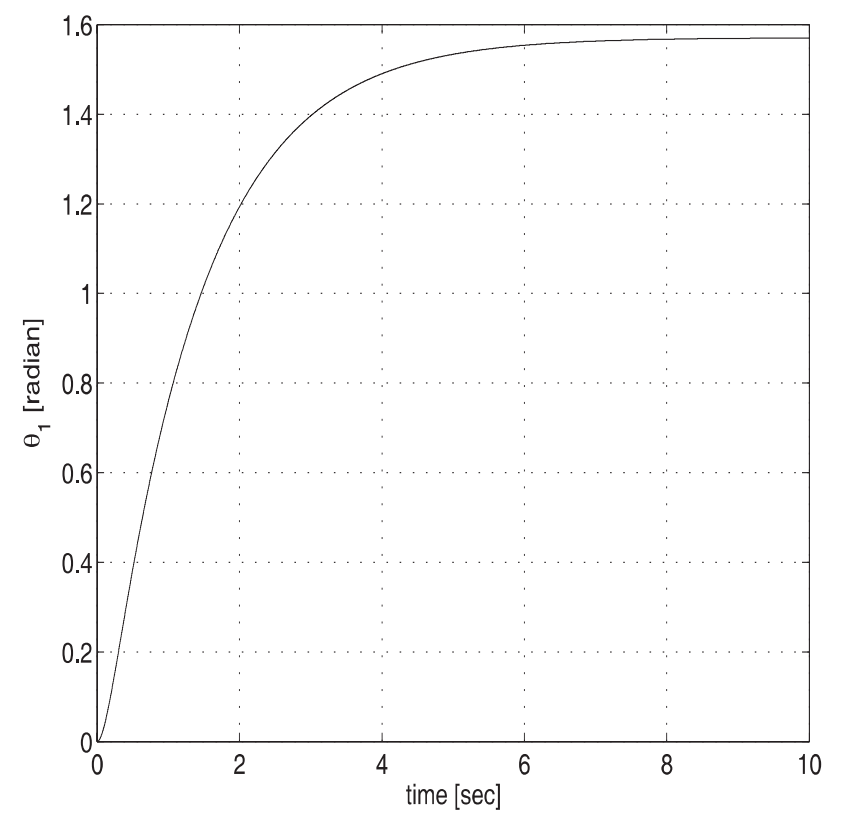

Figure 10. Joint angle of the link 1 - PDE model case 2.

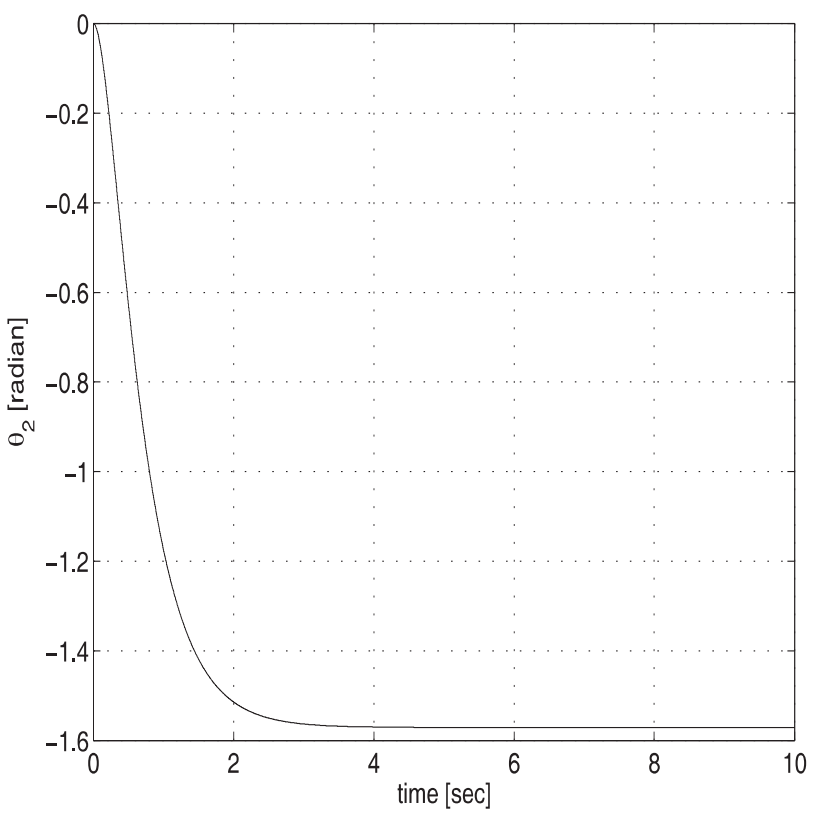

Figure 11. Joint angle of the link 2-PDE model case 2. 


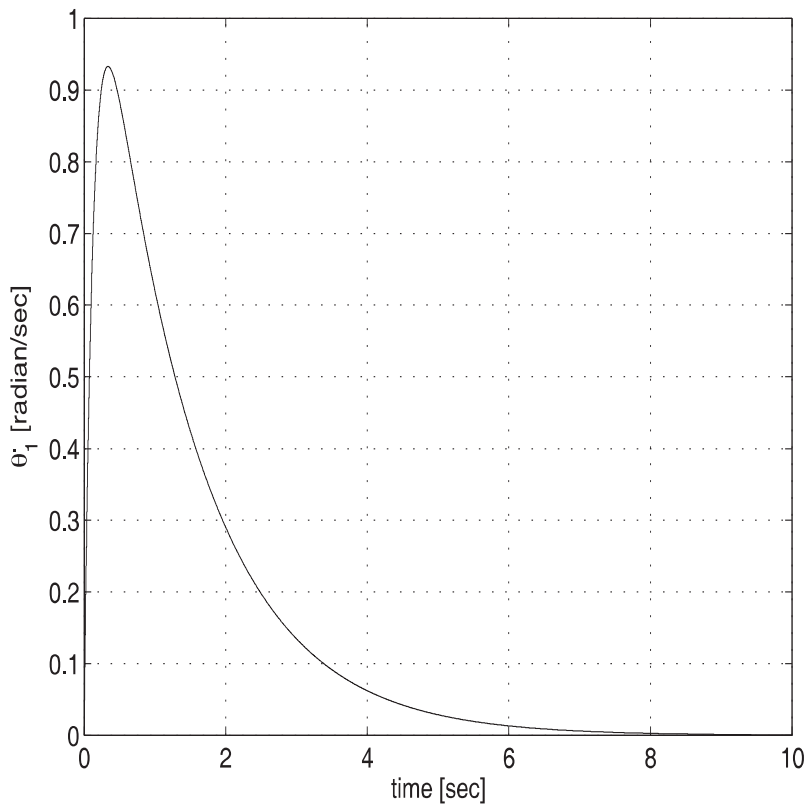

Figure 12. Joint angular velocity of the link $1-$ PDE model case 2.

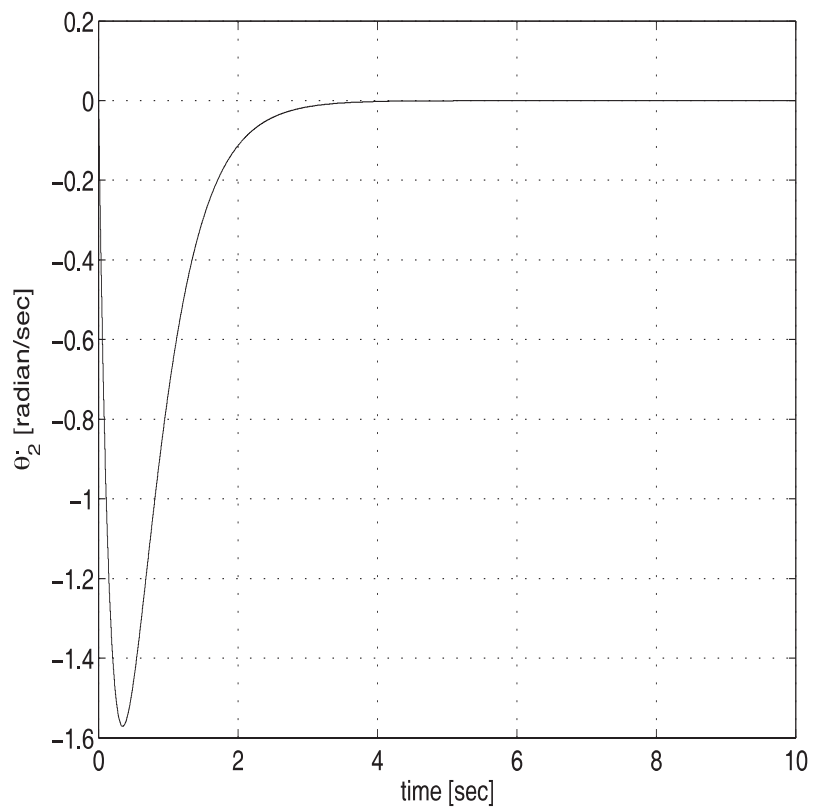

Figure 13. Joint angular velocity of the link $2-$ PDE model case 2. 
636 M. DOG̃AN and Ö. MORGÜL

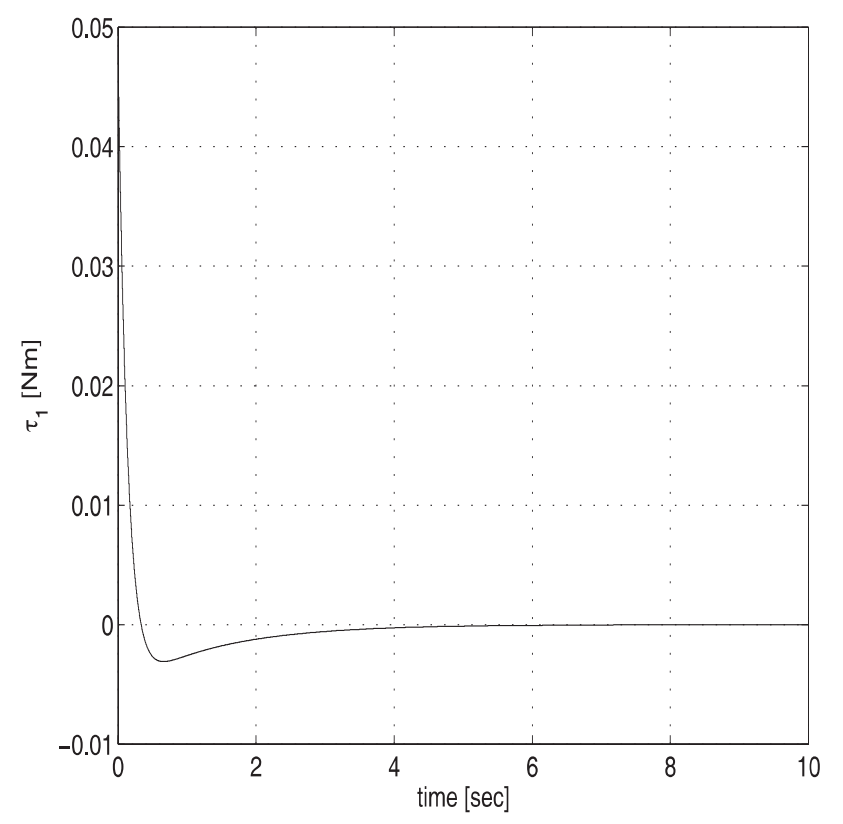

Figure 14. Control torque of joint 1 - PDE model case 2.

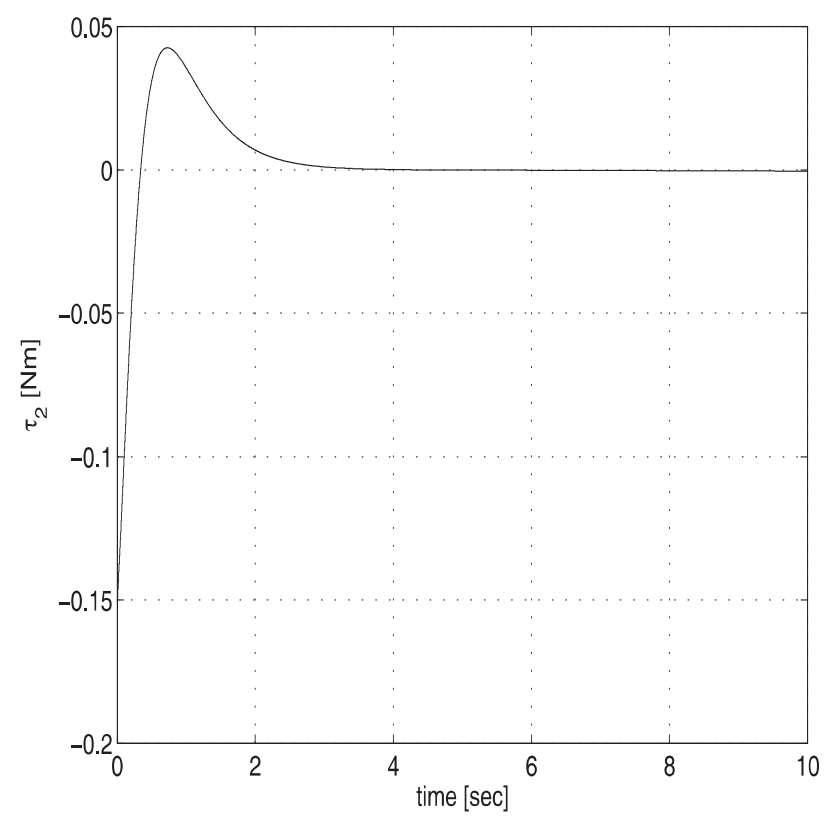

Figure 15. Control torque of joint 2 - PDE model case 2. 
Comparing the time responses for the FEM case in Dogan and Istefanopulos (2007) with the simulation results for PDE cases, it is observed that the required control energy in the PDE cases is much less than the one in the FEM due to the exactness of the PDEs. Also, PDE responses are smoother than the ones in the FEM approach, and have no overshoot or no chattering for all states. However, the FEM approach may yield high performance for real-time controller implementation, and is known to be extendible to the multi-link case easily; see e.g. Dogan and Istefanopulos (2007) and also Remark 2.1.

Remark 4.1. Numerical setup, tapering and controller parameters are given in Dogan (2006). Link geometry, mass and inertia parameters are given in Zhang's experimental work; see e.g. Zhang et al. (2005). We assumed that the links are aluminium alloy; hence, the Young's modulus and densities are chosen accordingly; see e.g. Hibbeler (2003).

Remark 4.2. We first note that experimental results on multi-link flexible systems are quite few in the literature due to the very complex nature of both the theoretical analysis and practical problems; notable exceptions are the works of Luo (1993), in which a single link is used, and Zhang et al. (2005), in which two links are used. The majority of the work on flexible structures which uses the PDE approach relies on numerical simulations for verification, as in our paper. We note that PDE approaches to model flexible structures are usually considered as better approximations to the physical nature of such systems as compared to ODE approaches; see e.g. Meirovitch (2001), Wang et al. (2003), Morgül (1991) and Guo (2002). Moreover, numerical simulations for their verification can be considered as a natural approach due to their complexity. We note that accurate simulation of a nonlinear PDE is itself a very complex problem and there are various works in the literature which are devoted to this subject; see e.g. Greenspan and Casulli (1988) and Chari and Salon (2000). Also, note that all of the techniques for the simulation of nonlinear PDEs (e.g. the FEM) utilize an appropriate conversion of PDEs to nonlinear ODEs and to the simulation of these ODEs. However, choosing discretization time and space steps requires special attention since they play an important role in getting meaningful simulation results; see e.g. Greenspan and Casulli (1988), Chari and Salon (2000) and Abhyankar et al. (1993). We also note that in this work we mainly consider nonuniform cross sections for the flexible links. Despite the fact that such links have some advantages over uniform links, they are seldom considered in the literature due to the resulting theoretical difficulties. One important reason to utilize a nonuniform flexible link is the fact that it increases the system bandwidth; see e.g. Moallem et al. (2000) and Wang et al. (1992). As a consequence, this may increase the system performance (e.g. it may result in a smaller settling time). The controller proposed in our work is, in our opinion, simpler in form than the ones proposed in similar works; see e.g. Zhang et al. (2005). Also, note that while at the ends of the links the strain feedback is used in the literature, see e.g. Zhang et al. (2005), in addition to these terms we also utilize accelerations. In our opinion, such terms could be measured effectively with newly developed high-performance accelerometers. Finally, while the damping terms are introduced in most of the models considered in the literature, see e.g. Zhang et al. (2004) and Zhang et al. (2005), such terms are not included in our model. 


\section{CONCLUSIONS}

In this paper, we considered both the modeling and the control of a two-link flexible arm with variable cross section. The physical configuration which we considered is given in Figure 1. For the modeling, we used the PDE approach which is known to yield more accurate results as compared to finite-dimensional, e.g. ODE or FEM, approaches. The basic equations of motion are obtained by using Hamilton's principle. Then, we designed a control law based on the Lyapunov approach and stability proofs are based on LaSalle's invariance principle extended to the infinite-dimensional case; see e.g. Luo et al. (1999). We note that, in the proof of our main stability result, the infinite dimensionality of the system considered in this paper has been retained as opposed to other energy-based approaches used for the multilink robot arms in the literature. In the majority of these latter works, a finite-dimensional ODE approximation of the original PDE is used and this approach is known to have many disadvantages; see Remark 2.1. We also presented some simulation examples, which show the robustness of our method with respect to parameter variations.

The present work could be extended in various ways. One natural extension is to consider PDE modeling of a multi-link flexible arm case. However, such an extension by using the PDE approach might result in a highly complex set of equations. Robustness results could also be proven more rigorously. Also, adaptation of the proposed controller with respect to external unknown disturbances may be a possible extension. However, these points require and deserve further investigation.

\section{A. PROOF OF DECOUPLING}

If the dominant part of the control laws 16-17 is substituted into equations $4-5$, then we obtain

$$
\ddot{\theta}_{i}=-\frac{\ddot{w}_{i}\left(l_{i}, t\right)}{l_{i}}-K_{i}\left(\alpha \dot{\theta}_{i}+\beta\left(\theta_{i}-\theta_{i d}\right)\right),
$$

where $i=1,2$. Let us define $\theta_{e}=\theta_{i}-\theta_{i d}$; then we observe that $\dot{\theta}_{e}=\dot{\theta}_{i}, \ddot{\theta}_{e}=\ddot{\theta}_{i}$. Applying the Laplace transform to equation 41 with the zero initial conditions, we then obtain

$$
\begin{aligned}
\theta_{i}(s)-\frac{\theta_{i d}}{s} & =-\frac{\mathcal{L}\left\{\ddot{w}_{i}\left(l_{i}, t\right)\right\}}{K_{i} \beta l_{i}} \frac{K_{i} \beta}{\left(s^{2}+K_{i} \alpha s+K_{i} \beta\right)}, \\
\theta_{i}(s)-\frac{\theta_{i d}}{s} & =-\frac{\mathcal{L}\left\{\ddot{w}_{i}\left(l_{i}, t\right)\right\}}{K_{i} \beta l_{i}} L_{p}(s),
\end{aligned}
$$

where $i=1,2$ and $L_{p}(s)$ is the well-known second-order low-pass filter transfer function with the natural frequency $w_{n i}=\sqrt{K_{i} \beta}$ and the damping ratio $\zeta_{i}=\alpha \sqrt{K_{i} / 4 \beta}$. Then, the bandwidth of the low-pass filter can be calculated, see e.g. Kuo (1992) and Kuo (1991), such that 


$$
w_{B i}=w_{n i}\left[\left(1-2 \zeta_{i}^{2}\right)+\sqrt{4 \zeta_{i}^{4}-4 \zeta_{i}^{2}+2}\right]^{\frac{1}{2}},
$$

where $w_{B i}$ can be adjusted to a small value less than $1 / 5$ of the fundamental vibration frequency by choosing the convenient controller parameters $\left(K_{i}, \alpha, \beta\right)$. The minimum fundamental vibration frequency for both links can be approximated as, see e.g. Guo (2002) and Hartog (1985),

$$
w_{\mathrm{fmin}}=\sqrt{\frac{3.03 E I_{\min }}{l_{2}^{3} m_{t 2}}} .
$$

This formula has been verified in the FEM approach for nonlinear cases and e.g. $w_{\text {fmin }}=$ $9.7 \mathrm{rad} / \mathrm{sec}$, and also $w_{B 1}=1.8 \mathrm{rad} / \mathrm{sec}$ and $w_{B 2}=1.6751 \mathrm{rad} / \mathrm{sec}$ are designed for simulation studies with the parameters in Table 1 . Note that $\ddot{w}_{i}\left(l_{i}, t\right)$ will have the same harmonics with the vibration modes due to undamped equations 2 and 3; see Remark 4.2. Therefore, its frequency spectrum cannot include lower frequency harmonics than the fundamental one and starts with $w_{\text {fmin }}$; see e.g. Guo (2002, Proposition 2.3) and also Meirovitch (2001, Ch. 8). Hence, the amplitude of the right-hand side of equation 42 becomes zero. Thus, we get $\theta_{i}-\theta_{i d}=0$ by inverse Laplace transformation of the left-hand side of equation 42 and hence $\dot{\theta}_{i}=0$ as well. Consequently, equation 41 can be simplified further, such as

$$
\ddot{\theta}_{i}=-\frac{\ddot{w}_{i}\left(l_{i}, t\right)}{l_{i}} .
$$

Since $\ddot{\theta}_{i}, \ddot{w}_{i}, \dot{\theta}_{i}$ and $\dot{w}_{i}$ are continuous in time with zero initial conditions, time integration of equation 43 will give the following result:

$$
\dot{\theta}_{i}=-\frac{\dot{w}_{i}\left(l_{i}, t\right)}{l_{i}} .
$$

Applying equations 43 and 44 to the system 2-10, we obtain

$$
\begin{aligned}
\ddot{w}_{1}+\frac{\left(E I_{1}\left(x_{1}\right) w_{1 x x}\right)_{x x}}{\rho_{1}\left(x_{1}\right)} & =-x_{1} \ddot{\theta}_{1}, \\
\ddot{w}_{2}+\frac{\left(E I_{2}\left(x_{2}\right) w_{2 x x}\right)_{x x}}{\rho_{2}\left(x_{2}\right)} & =-x_{2} \ddot{\theta}_{2}, \\
l_{1} \ddot{\theta}_{1} & =-\ddot{w}_{1}\left(l_{1}, t\right), \\
l_{2} \ddot{\theta}_{2} & =-\ddot{w}_{2}\left(l_{2}, t\right), \\
-\tau_{b 2}+\tau_{e 1}-I_{h 2}\left[\ddot{\theta}_{1}+\ddot{\theta}_{2}\right]-I_{t 1}\left[\ddot{\theta}_{1}+\ddot{w}_{1 x}\left(l_{1}, t\right)\right] & =0, \\
{\left[\left(E I_{2}\left(x_{2}\right) w_{2 x x}\right)_{x}\right]_{x_{2}=l_{2}} } & =0,
\end{aligned}
$$


where the main equations 2-5 are replaced with equations 45-48 and the new set of boundary conditions will be equations 49,50 and 10 . This new system 45-48, with very simple boundary conditions, is linear and decoupled, as shown above.

\section{B. PROOF OF ASYMPTOTIC STABILITY}

In order to initiate the stability analysis, equations 2-5 should be rewritten in the new variables as follows:

$$
\begin{aligned}
\mathbf{z} & =\left[\begin{array}{llllllll}
z_{1} & z_{2} & z_{3} & z_{4} & z_{5} & z_{6} & z_{7} & z_{8}
\end{array}\right]^{T} \\
& =\left[\begin{array}{lllllllll}
w_{1} & \dot{w}_{1} & w_{2} & \dot{w}_{2} & \theta_{1}-\theta_{1 d} & \dot{\theta}_{1} & \theta_{2}-\theta_{2 d} & \dot{\theta}_{2}
\end{array}\right]^{T} .
\end{aligned}
$$

The system 2-5 can be decomposed to linear and nonlinear parts separately, see Morgül (1991), such as

$$
\dot{\mathbf{z}}=A \mathbf{z}+f(\mathbf{z})
$$

where $A$ represents an infinite-dimensional linear operator and $f$ represents a nonlinear operator. For the definitions, the notation and the terminology used in this section, the reader is referred to e.g. Luo et al. (1999).

We first define the following Hilbert space:

$$
\begin{aligned}
H_{E}^{2}\left(0, l_{i}\right) & =\left\{w_{i} \in H^{2}\left(0, l_{i}\right) \mid w_{i}(0)=w_{i x}(0)=0\right\} \\
\mathbf{H}_{i} & =H_{E}^{2}\left(0, l_{i}\right) \times L^{2}\left(0, l_{i}\right)
\end{aligned}
$$

Next, we define an inner product induced norm as

$$
\left\|\left(w_{i}, g_{i}\right)\right\|_{\mathbf{H}_{i}}^{2}=\int_{0}^{l_{i}}\left[\rho_{i}(x)\left|g_{i}(x)\right|^{2}+E I_{i}(x)\left|w_{i x x}(x)\right|^{2}\right] d x
$$

for $i=1,2$ and for all $\left(w_{i}, g_{i}\right) \in \mathbf{H}_{i}$; see e.g. see e.g. Guo (2002). Let us define the operator $A_{i}: D\left(A_{i}\right)\left(\subset \mathbf{H}_{i}\right) \rightarrow \mathbf{H}_{i}$ as

$$
\begin{aligned}
A_{i}\left(w_{i}, g_{i}\right) & =\left(g_{i}, \frac{-1}{\rho_{i}(x)}\left(E I_{i}(x) w_{i x x}(x)\right)_{x x}\right), \\
D\left(A_{i}\right) & =\left\{\left(w_{i}, g_{i}\right) \in\left(H_{E}^{2} \cap H^{4}\right) \times H_{E}^{2}\right\},
\end{aligned}
$$

where $A_{i}^{-1}$ is a compact operator on $\mathbf{H}_{i}$; see e.g. Guo (2002, Lemma 2.1). We also define the function spaces $\widehat{\mathbf{H}}$ and $\widehat{\mathbf{D}}$ as follows:

$$
\widehat{\mathbf{H}}=\mathbf{H}_{1} \times \mathbf{H}_{2} \times \mathbf{R} \times \mathbf{R} \times \mathbf{R} \times \mathbf{R},
$$




$$
\widehat{\mathbf{D}}=D\left(A_{1}\right) \times D\left(A_{2}\right) \times \mathbf{R} \times \mathbf{R} \times \mathbf{R} \times \mathbf{R} .
$$

By using the set of equations 2-10 and integration by parts, we can express the linear operator $A: \widehat{\mathbf{H}} \rightarrow \widehat{\mathbf{H}}$ and the nonlinear operator $f: \widehat{\mathbf{H}} \rightarrow \widehat{\mathbf{H}}$ in equation 53 as follows:

$$
\begin{gathered}
A=\left[\begin{array}{ccc}
A_{1} & 0_{2 \times 2} & 0_{1 \times 4} \\
0_{2 \times 2} & A_{2} & B_{1} \\
0_{4 \times 2} & 0_{4 \times 2} & B_{2}
\end{array}\right], \\
f(\mathbf{z})=\left[\begin{array}{c}
0 \\
-x_{1} W_{1}-\frac{x_{1}}{I_{t 1}}\left(\tau_{e 1}-\tau_{b 2}+\tau_{e 2}\right) \\
0 \\
-x_{2} W_{2}+\frac{x_{2}}{I_{t 1}}\left(\tau_{e 1}-\tau_{b 2}\right)+x_{2} \frac{I_{h 2}+I_{t 1}}{I_{h 2} I_{t 1}} \tau_{e 2}+f_{0} \\
0 \\
W_{1}+\frac{1}{I_{t 1}}\left(\tau_{e 1}-\tau_{b 2}+\tau_{e 2}\right) \\
0 \\
W_{2}-\frac{1}{I_{t 1}}\left(\tau_{e 1}-\tau_{b 2}\right)-\frac{I_{h 2}+I_{t 1}}{I_{h 2} I_{t 1}} \tau_{e 2}
\end{array}\right],
\end{gathered}
$$

where $A_{1}, A_{2}$ are given in equation 57; $\tau_{e i}, \tau_{b i}$ are defined by equations 11 and 12 and $B_{1}, B_{2}, W_{1}, W_{2}, f_{0}$ are given below:

$$
\begin{aligned}
& B_{1}=\left[\begin{array}{cccc}
0 & 0 & \frac{x_{1} I_{h 2} \widetilde{\beta}}{I_{h 2}+I_{t 1}} & \frac{x_{1} I_{h 2} \widetilde{\alpha}}{I_{h 2}+I_{t 1}} \\
0 & 0 & 0 & 0 \\
0 & 0 & -x_{2} \widetilde{\beta} & -x_{2} \widetilde{\alpha}
\end{array}\right], \\
& B_{2}=\left[\begin{array}{cccc}
0 & 1 & 0 & 0 \\
0 & 0 & \frac{-I_{h 2} \widetilde{\beta}}{I_{h 2}+I_{t 1}} & \frac{-I_{h 2} \widetilde{\alpha}}{I_{h 2}+I_{t 1}} \\
0 & 0 & 0 & 1 \\
0 & 0 & \widetilde{\beta} & \widetilde{\alpha}
\end{array}\right], \\
& W_{1}=\left.\frac{I_{h 2}}{\gamma_{2} I_{t 1}} \frac{\left(E I_{2}\left(x_{2}\right) w_{2 x x}\right)_{x x}}{l_{2} \rho_{2}\left(x_{2}\right)}\right|_{x_{2}=l_{2}},
\end{aligned}
$$




$$
\begin{aligned}
W_{2} & =\left.\frac{I_{h 2}+I_{t 1}}{-\gamma_{2} I_{t 1}} \frac{\left(E I_{2}\left(x_{2}\right) w_{2 x x}\right)_{x x}}{l_{2} \rho_{2}\left(x_{2}\right)}\right|_{x_{2}=l_{2}}, \\
f_{0} & =\cos \theta_{2}\left[K_{1} l_{1}\left(\alpha \dot{\theta}_{1}+\beta\left(\theta_{1}-\theta_{1 d}\right)\right)\right. \\
& \left.-\frac{\gamma_{1} l_{1}}{I_{h 1}}\left(\tau_{b 2}-\tau_{e 2}-K \dot{\theta}_{1}\right)\right]+\dot{\theta}_{2} \sin \theta_{2}\left[\dot{w}_{1}\left(l_{1}, t\right)+l_{1} \dot{\theta}_{1}\right], \\
\widetilde{\beta} & =\frac{I_{h 2}+I_{t 1}}{\gamma_{2} I_{t 1}} K_{2} \beta, \widetilde{\alpha}=\frac{I_{h 2}+I_{t 1}}{\gamma_{2} I_{t 1}} K_{2} \alpha .
\end{aligned}
$$

We note that $B_{1}, B_{2}$ are finite-dimensional linear bounded operators, $f(\mathbf{0})=0$ and $f(\mathbf{z})$ is a differentiable function on $\widehat{\mathbf{H}}$.

Theorem B.1. Consider the system given by equation 53, where various operators, functions and spaces are defined in equations 51-69. Then,

$i$. The operator A generates a $C_{0}$ semigroup $T(t)$ in $\widehat{\mathbf{H}}$.

ii. For any $z(0) \in \widehat{\mathbf{D}}$, there exists a unique (classical) solution $z(t)$ of equation 53 for $t \geq 0$; moreover, $z(t) \in \widehat{\mathbf{D}}$.

iii. For any $z(0) \in \widehat{\mathbf{D}}$, we have $\lim _{t \rightarrow \infty} z(t)=0$, i.e. the closed-loop system is asymptotically stable.

Proof: we note that for the standard terminology on semigroups, the reader is referred to e.g. Luo et al. (1999).

i: It can be easily shown that $A_{i}$ generates a $C_{0}$ semigroup $T(t)$ in $\mathbf{H}_{i}, i=1,2$; see e.g. Guo (2002)) and Luo et al. (1999). Since $B_{1}$ and $B_{2}$ are bounded linear operators, it then easily follows that $A$ generates a $C_{0}$ semigroup $T(t)$ in $\widehat{\mathbf{H}}$; see e.g. Luo et al. (1999).

ii: Since $f(\cdot)$ is a differentiable function on $\widehat{\mathbf{H}}$, it follows easily from i that, for any $z(0) \in$ $\widehat{\mathbf{D}}$, there exists a $T_{e}>0$ such that equation 53 has a unique (classical) solution $z(t) \in \widehat{\mathbf{D}}$ for $t \in\left[0, T_{e}\right)$. However, since by equation 39 the solutions are bounded, it follows easily that local solutions can be extended to global solutions, i.e. we can take $T_{e}=\infty$; see e.g. Luo et al. (1999).

iii: Since $A_{i}^{-1}$ are compact operators on $\mathbf{H}_{i}, i=1,2$, see e.g. Guo (2002), and since $B_{1}$ and $B_{2}$ are bounded operators, it follows easily that $(\lambda I-A)^{-1}$ is a compact operator on $\widehat{\mathbf{H}}$ for sufficiently large $\lambda>0$ (e.g. for $\lambda>\widetilde{\alpha}$ ); see e.g. Guo (2002) and Luo et al. (1999). It then follows that the bounded solutions are also precompact in $\widehat{\mathbf{D}} \subset \widehat{\mathbf{H}}$; see e.g. Luo et al. (1999, Theorem 3.65, p. 162). It then follows that the $\omega$-limit set $\omega(z)$ of $z(t)$ is nonempty, compact, invariant and $z(t) \rightarrow \omega(z)$ as $t \rightarrow \infty$; moreover, $\omega(z)$ is the smallest set satisfying this property; see e.g. Luo et al. (1999, Theorem 3.61, p. 159). Moreover, since $\dot{V}(t) \rightarrow 0$ as $t \rightarrow \infty$, see equation 40, we also have $z(t) \rightarrow \widehat{\mathbf{C}}$ as $t \rightarrow \infty$, where 


$$
\widehat{\mathbf{C}}=\{\mathbf{z} \in \widehat{\mathbf{D}} \mid \dot{V}(\mathbf{z})=0\}
$$

see e.g. Khalil (2002, Theorem 8.4, p. 323) and the arguments following the proof. By combining these, we conclude that $z(t) \rightarrow \widehat{\Omega} \subset \widehat{\mathbf{C}}$, where $\widehat{\Omega}$ is the largest invariant set in $\widehat{\mathbf{C}}$. Now, as in the standard application of LaSalle's invariance principle, to complete the proof, we should show that $\dot{V}=0$ implies that $\mathbf{z}=0$. If $\dot{V}=0$ then $\delta W_{n c}=0$, since the power associated with nonconservative forces is equal to the time rate of change of the total energy; see e.g. Meirovitch (2001, Section 1.3). For the system 2-10, we have

$$
\delta W_{n c}=\tau_{1} \delta \theta_{1}+\tau_{2} \delta\left(\theta_{2}-w_{1 x}\left(l_{1}, t\right)\right) .
$$

Since $\delta \theta_{1}$ and $\delta\left(\theta_{2}-w_{1 x}\left(l_{1}, t\right)\right)$ are arbitrary nonzero variations, we obtain $\tau_{1}=0$ and $\tau_{2}=0$. On the other hand, from the calculations given in Section 3, obviously we have $\dot{\theta}_{i}=\ddot{\theta}_{i}=0, i=1,2$, on the $\omega$-limit set; see e.g. equations 25, 30-32. Hence, from equations 4, 22, 5 and 49, we obtain $\tau_{b i}=\tau_{e i}=0, i=1,2$, on the $\omega$-limit set; see equation 11. Similarly, by using these results, Appendix A and from equations 7 and 8 , we obtain

$$
\left[\left(E I_{i}\left(x_{i}\right) w_{2 x x}\right)_{x}\right]_{x_{i}=l_{i}}=0, \quad i=1,2 .
$$

Hence, on the $\omega$-limit set equations 2 and 3 become

$$
\begin{aligned}
& \rho_{1}\left(x_{1}\right) \ddot{w}_{1}+\left(E I_{1}\left(x_{1}\right) w_{1 x x}\right)_{x x}=0, \\
& \rho_{2}\left(x_{2}\right) \ddot{w}_{2}+\left(E I_{2}\left(x_{2}\right) w_{2 x x}\right)_{x x}=0 .
\end{aligned}
$$

It follows from the standard results that the solutions $w_{i}(x, t)$ of equations 73 and 74 can be expressed by using separation of variables as

$$
w_{i}(x, t)=\phi_{i}(x) e^{\lambda_{i} t}, \quad i=1,2
$$

see e.g. Baruh (1999, Section 11.4). Here, the $\lambda_{i}$ are nonzero complex eigenvalues and the $\phi_{i}$ are eigenfunctions for equations 73 and 74. By using equation 75 in equations 73 and 74 , we obtain

$$
\begin{aligned}
& \lambda_{1}^{2} \rho_{1}\left(x_{1}\right) \phi_{1}\left(x_{1}\right)+\frac{d}{d x_{1}^{2}}\left(E I_{1}\left(x_{1}\right) \frac{d}{d x_{1}^{2}} \phi_{1}\left(x_{1}\right)\right)=0 \\
& \lambda_{2}^{2} \rho_{2}\left(x_{2}\right) \phi_{2}\left(x_{2}\right)+\frac{d}{d x_{2}^{2}}\left(E I_{2}\left(x_{2}\right) \frac{d}{d x_{2}^{2}} \phi_{2}\left(x_{2}\right)\right)=0 ;
\end{aligned}
$$

see e.g. Baruh (1999). One can define $\phi_{i}(x)$ as

$$
\phi_{i}(x)=p_{i}(x) r_{i}(x),
$$


where $p_{i}(x)=a_{i} x^{4}+b_{i} x^{3}+c_{i} x^{2}+d_{i} x+e_{i}$ and $r_{i}(x)$ is a possibly nonlinear function which is fourth-order differentiable at least and satisfies the conditions such that $r_{i}(0) \neq 0, r_{i}(l i) \neq 0$. By taking the derivatives, we obtain

$$
\begin{aligned}
\phi_{i x}(x) & =p_{i x}(x) r_{i}(x)+p_{i}(x) r_{i x}(x), \\
\phi_{i x x}(x) & =p_{i x x}(x) r_{i}(x)+2 p_{i x}(x) r_{i x}(x)+p_{i}(x) r_{i x x}(x), \\
\phi_{i x x x}(x) & =p_{i x x x}(x) r_{i}(x)+3 p_{i x x}(x) r_{i x}(x)+3 p_{i x}(x) r_{i x x}(x)+p_{i}(x) r_{i x x x}(x) .
\end{aligned}
$$

By using the boundary conditions in the $\omega$-limit set as given above, we obtain

$$
\begin{aligned}
\phi_{i}(0) & =0 \text { from equation } 10, \\
\phi_{i x}(0) & =0 \text { from equation } 10, \\
\phi_{i x x}(0) & =0 \text { from } \tau_{b i}=0, \\
\phi_{i}\left(l_{i}\right) & =0 \text { from equations } 45 \text { to } 48,76 \text { and } 77, \\
\phi_{i x}\left(l_{i}\right) & =0, \quad \text { see } \text { Remark } 2.2, \\
\phi_{i x x}\left(l_{i}\right) & =0 \text { from } \tau_{e i}=0, \\
\phi_{i x x x}\left(l_{i}\right) & =0 \text { from equation } 72 .
\end{aligned}
$$

Note that the first three equations given above imply that $c_{i}=d_{i}=e_{i}=0$. Then, by using any two of the remaining equations, one can easily obtain $a_{i}=b_{i}=0$. Hence, we have $\phi_{i}(x)=0$. From equation 75 it then follows easily that the only possible solution of equations 73 and 74 on the $\omega$-limit set is the trivial solution, i.e. $w_{i}(x, t)=0$. By combining this result with equations $25,30-32$, we conclude that the only possible solution of equation 53 on the $\omega$-limit set is the trivial solution, i.e. $z=0$. The asymptotic stability result now follows from LaSalle's invariance principle; see e.g. Luo et al. (1999, Theorems 3.61 and 3.64).

\section{KINETIC AND POTENTIAL ENERGIES}

In order to derive the PDE model, kinetic and potential energy expressions for the robot arm are given below (Zhang et al., 2005):

$$
T_{1}=\frac{1}{2} \int_{0}^{l_{1}} \rho_{1}\left(x_{1}\right)\left[x_{1} \dot{\theta}_{1}+\dot{w}_{1}\right]^{2} d x_{1},
$$




$$
\begin{aligned}
T_{2} & =\frac{1}{2} \int_{0}^{l_{2}} \rho_{2}\left(x_{2}\right)\left\{\left[l_{1} \dot{\theta}_{1}+\dot{w}_{1}\left(l_{1}, t\right)\right] \cos \left(\theta_{2}\right)+x_{2} \dot{\theta}_{2}+\dot{w}_{2}\right\}^{2} d x_{2} \\
& +\frac{1}{2} \int_{0}^{l_{2}} \rho_{2}\left(x_{2}\right)\left\{\left[l_{1} \dot{\theta}_{1}+\dot{w}_{1}\left(l_{1}, t\right)\right] \sin \left(\theta_{2}\right)\right\}^{2} d x_{2}, \\
T_{3} & =\frac{1}{2} I_{h 1} \dot{\theta}_{1}^{2}, \\
T_{4} & =\frac{1}{2} I_{t 1}\left(\dot{\theta}_{1}+\dot{w}_{1 x}\left(l_{1}, t\right)\right)^{2}+\frac{1}{2} m_{t 1}\left[l_{1} \dot{\theta}_{1}+\dot{w}_{1}\left(l_{1}, t\right)\right]^{2}, \\
T_{5} & =\frac{1}{2} I_{h 2}\left(\dot{\theta}_{1}+\dot{\theta}_{2}\right)^{2}+\frac{1}{2} m_{h 2}\left[l_{1} \dot{\theta}_{1}+\dot{w}_{1}\left(l_{1}, t\right)\right]^{2}, \\
T_{6} & =\frac{1}{2} I_{t 2}\left(\dot{\theta}_{1}+\dot{\theta}_{2}+\dot{w}_{2 x}\left(l_{2}, t\right)\right)^{2}+\frac{1}{2} m_{t 2}\left\{\left[l_{1} \dot{\theta}_{1}+\dot{w}_{1}\left(l_{1}, t\right)\right] \sin \left(\theta_{2}\right)\right\}^{2} \\
& +\frac{1}{2} m_{t 2}\left\{\left[l_{1} \dot{\theta}_{1}+\dot{w}_{1}\left(l_{1}, t\right)\right] \cos \left(\theta_{2}\right)+l_{2} \dot{\theta}_{2}+\dot{w}_{2}\left(l_{2}, t\right)\right\}^{2},
\end{aligned}
$$

where $T_{1}$ and $T_{2}$ are the kinetic energies of link 1 and link 2, respectively; $T_{3}$ is the kinetic energy of hub 1 or the base joint; $T_{4}$ is the kinetic energy of the tip mass at the end of link 1 ; $T_{5}$ is the kinetic energy of hub $2 ; T_{6}$ is the kinetic energy of the tip mass at the end of link 2 and the total strain potential energy can be expressed as (Zhang et al., 2005)

$$
V_{s}=\frac{1}{2} \int_{0}^{l_{1}} E I_{1}\left(x_{1}\right) w_{1 x x}^{2} d x_{1}+\frac{1}{2} \int_{0}^{l_{2}} E I_{2}\left(x_{2}\right) w_{2 x x}^{2} d x_{2} \text {. }
$$

\section{REFERENCES}

Abhyankar, N. S., Hall II, E. K., and Hanagud, S. V., 1993, "Chaotic vibrations of beams: numerical solution of partial differential equations," ASME Journal of Applied Mechanics 60, 167-174.

Baruh, H., 1999, Analytical Dynamics, McGraw-Hill, Boston, MA.

Chari, M. V. K. and Salon, S. J., 2000, Numerical Methods in Electromagnetism, Academic Press, San Diego, CA.

Dog̃an, M., 2006, “Optimal nonlinear controller design for flexible robot manipulators,” Ph.D. thesis, Bog̃aziçi University, Istanbul.

Dog̃an, M. and Istefanopulos, Y., 2007, “Optimal nonlinear controller design for flexible robot manipulators with adaptive internal model," IET Control Theory and Applications 1(3), 770-778.

Greenspan, D. and Casulli, V., 1988, Numerical Analysis for Applied Mathematics, Science and Engineering, AddisonWesley, Reading, MA.

Guo, B. Z., 2002, "Riesz basis property and exponential stability of controlled Euler-Bernoulli beam equations with variable coefficients," SIAM Journal on Control and Optimization 40(6), 1905-1923.

Den Hartog, J. P., 1985, Mechanical Vibrations, Dover, New York.

Hastings, G. G. and Book, W. J., 1987, “A linear dynamic model for flexible robotic manipulators," IEEE Control System Magazine 7, 61-64.

Hattori, M., Tadokoro, S., and Takomori, T., 1997, "A generalization of direct strain feedback control for flexible structures with spatially varying parameters and a tip body," in Proceedings of the 16th American Control Conference, Albuquerque, NM, USA, pp. 2333-2336. 
Hibbeler, R. C., 2003, Mechanics of Materials, Prentice-Hall, Upper Saddle River, NJ.

Khalil, H. K., 2002, Nonlinear Systems, 3rd edn, Prentice-Hall, Upper Saddle River, NJ.

Kuo, B. C., 1991, Automatic Control Systems, 6th edn, Prentice-Hall, Upper Saddle River, NJ.

Kuo, B. C., 1992, Digital Control Systems, 2nd edn, Oxford University Press, New York.

Luo, Z. H., 1993, "Direct strain feedback control of flexible robot arms: new theoretical and experimental results," IEEE Transactions on Automatic Control 38(11), 1610-1622.

Luo, Z. H., Guo, B. Z., and Morgül, Ö., 1999, Stability and Stabilization of Infinite Dimensional Systems with Applications, Springer, London.

Meirovitch, L., 2001, Fundamentals of Vibrations, McGraw-Hill, New York.

Moallem, M., Patel, R. V., and Khorasani, K., 2000, "Optimization of an actuated flexible arm for improved control properties," in Proceedings of the IEEE International Conference on Control Applications, Anchorage, AK, September 25-27, pp. 709-714.

Morgül, Ö., 1991, “Orientation and stabilization of a flexible beam attached to a rigid body: planar motion," IEEE Transactions on Automatic Control 36(8), 953-962.

Raab, F. J. and Trudnowski, D. J., 1998, "Experiments in two-axis vibration damping using inertial torques through momentum wheel control," in Proceedings of the American Control Conference, Philadelphia, PA, June 24-26, pp. 3477-3481.

Reddy, J. N., 1993, An Introduction to the Finite Element Method, McGraw-Hill, New York.

Rokui, M. R. and Khorasani, K., 2000, "Experimental results on discrete-time nonlinear adaptive tracking control of a flexible-link manipulator," IEEE Transactions on Systems, Man, and Cybernetics 30(1), 151-164.

Siciliano, B. and Villani, L., 2001, "Two-time scale force and position control of flexible manipulators," in Proceedings of the IEEE International Conference on Robotics and Automation, Seoul, Korea, May 21-26, pp. 2729-2734.

Vandegrift, M. W., Lewis, F. L., and Zhu, S. Q., 1994, "Flexible-link robot arm control by a feedback linearization/singular perturbation approach,” Journal of Robotic Systems 11(7), 591-603.

Wang, F. Y. and Russell, J. L., 1992, "Optimum shape construction of flexible manipulators with tip loads," in Proceedings of the 31st IEEE Conference on Decision and Control, Tucson, AZ, USA, December 16-18, pp. 311-316.

Wang, F. Y. and Gao, Y., 2003, Advanced Studies of Flexible Robotic Manipulators, World Scientific, NJ.

Zhang, X., Xu, W., and Nair, S. S., 2004, "Comparison of some modeling and control issues for a flexible two-link manipulator," ISA Transactions 43, 509-525.

Zhang, X., Xu, W., Nair, S. S., and Chellabonia, V., 2005, "PDE modeling and control of a flexible two-link manipulator," IEEE Transactions on Control Systems Technology 13(2), 301-312.

Zhang, X., Xu, W., Nair, S. S., and Chellabonia, V., 2002, "PDE modeling and control of a flexible two-link manipulator," in Proceedings of the 16th American Control Conference, Anchorage, AK, pp. 3796-3801.

Zhang, X., 2001, "Multi-scale system dynamics and control - two case studies," PhD dissertation, University of Missouri-Columbia, Columbia, MO.

Zhu, Ye, Qiu, Jinhao, and Tani, Junji, 2001, "Simultaneous optimization of a two-link flexible robot arm,” Journal of Robotic Systems 18(1), 29-38. 